\title{
Comparison of Mean Platelet Volume in Acute Myocardial Infarction vs. Normal Coronary Angiography
}

\author{
Alireza Rai ${ }^{1}$, Mohammadreza Saidi ${ }^{1}$, Nahid Salehi ${ }^{1}$, Farzad Sahebjamei ${ }^{1}$, Masoud Jalilian ${ }^{2}$ \& Parisa Janjani ${ }^{2,3}$ \\ ${ }^{1}$ Cardiology Department, Medical School, Kermanshah University of Medical Sciences, Kermanshah, Iran \\ ${ }^{2}$ Kermanshah University of Medical Sciences, Kermanshah, Iran \\ ${ }^{3}$ Faculty of Social Sciences, Razi University, Kermanshah, Iran \\ Correspondence: Mohammadreza Saidi, MD, Cardiology Department, Medical School, Kermanshah University \\ of Medical Sciences, Kermanshah, Iran. Tel: 98-831-836-2022. E-mail: mrsaidi@yahoo.com
}

Received: October 18, 2015 Accepted: April 5, 2016 Online Published: May 13, 2016

doi:10.5539/gjhs.v8n11p320 URL: http://dx.doi.org/10.5539/gjhs.v8n11p320

\begin{abstract}
Considering the importance of cardiovascular disease and the role that platelets have in thrombosis formation in the coronary arteries, this study was done in order to assess platelet-related indices in patients who suffered acute myocardial infarction (MI) and compare them with those who had normal coronary angiography results.In this descriptive-analytical study, 200 patients who were admitted to our university hospital due to chest pain were included. The patients were divided into five groups including ST-segment elevation MI (STEMI), non-STEMI, unstable angina (UA), stable angina (SA), and healthy subjects (as control group). Platelet-related indices including platelet count as well asmean platelet volume (MPV) was determined. For this purpose, blood samples were taken from the patients upon admission and platelet count and volume were measured within three hours of admission. There was no statistically significant difference regarding MPV between the study groups $(\mathrm{P}>0.05)$.
\end{abstract}

MPV did not have any role in diagnosing various types of coronary artery disease (CAD).

Keywords: Mean platelet volume, acute coronary syndrome, stable angina, ST-segment elevation myocardial infarction (STEMI)

\section{Introduction}

Platelets are derived from megakaryocytes. Normal platelet count ranges from 140,000 to 440,000 per mL blood. Half-life of platelet is 7-10 days inside the circulation and one-third of platelets are found in the spleen. Mean platelet volume (MPV) is 6.8 to $10.5 \mathrm{fL}$ (Wintrobe's DICI elinicalHematolog, 2014). Increased platelet volume associated with high turnover is seen in Bernard-Soulier syndrome, increased platelet destruction, and proliferative disorders. Decreased platelet volume is seen in Big Spleen syndrome and sepsis. Platelets and coagulation factors like factor VII, fibrinogen, plasma viscosity, hematocrit, red blood cells (RBC), and white blood cells (WBC) are involved in the pathophysiology of atherosclerosis and have a non-separable role in acute thrombotic events. Also, platelets have an important role in instability of stable atherosclerotic plaques. When an injury occurs in the endothelial wall, the platelets become active by attachment to the subendothelial tissue. In this process, von-Willebrand factor also plays role and ultimately by platelet aggregation thromboxane-A2 granules as well as serotonin, adenosine, and other factors are released.

After platelet aggregation, plasma mediators (Epi and thrombin), platelet mediators, and extracellular/endothelial matrix strengthen this process. By release of platelet factors, more platelets are recruited and natural anti-thrombotic factors inhibit endothelial cells. Then, IIb/IIIareceptors expose which are the most frequent receptors on platelets (Harrison, 2012). Regarding the important role these receptors have in this process, they have been target for anti-platelet treatment. Finally, thrombus and platelet plug form.

Kiliçli-Camur (2005) in their study on 200 patients compared four groups of patients (including unstable angina (UA), stable angina (SA), ST-segment elevation myocardial infarction (MI), and non-STEMI) with control group (with normal coronary angiography results). It was reported that MPV was significantly higher in MI group than in SA or control groups. MPV was suggested as a risk factor (Kiliçli-Camur et al., 2009). In another study performed in 2009, the results demonstrated that high MPV is associated with decreased bleeding time (BT) and thromboxane concentration which reflects more activity of platelets with high volume (Viziolil, Muscari, \& 


\section{Muscari, 2009).}

In another study in 2008, MPV was described as an indicator for triage of patients with cardiac condition. In this study, 216 patients with acute coronary syndrome (ACS) were compared to 130 patients with SA. MPV was 10.4 $\mathrm{fL}$ in NSTEMI group, $10 \mathrm{fL}$ in UA, and $9.8 \mathrm{fL}$ in SA group. There were significant differences between groups (Yilmaze et al., 2008).

In another study in 2009, it was noted that platelet count in ACS patients $\left(210 \times 10^{3}\right)$ was lower than in SA patients $\left(267 \times 10^{3}\right)$ and control group $\left(256 \times 10^{3}\right)$, however MPV in ACS group $(10.67 \mathrm{fL})$ was higher than in SA group (10.93 fL) and control group (10.31 fL) (Ranjh et al, 2009).

In another research study in 2010, MPV was compared between diabetics and non-diabetics with coronary artery disease (CAD). MPV was significantly higher in diabetics (10.93 fL) compared to non-diabetics $(9.12 \mathrm{fL})$ (Tavil, Sen, \& Yazici, 2010).

Therefore, considering the mentioned studies, it seems that increased platelet activity is likely to be associated with severity of ischemic heart disease (IHD). Larger platelets are likely more active and have more ability for thrombogenesis compared to smaller platelets (ViziolilL, Muscari, \& Muscari, 2009; Yilmaze et al., 2008; Ranjh, 2009).

Therefore, there should be association between platelet size and events from platelet increased activity like IHD, for which MPV can be used. Also, from the results of the current study and other reports prediction can be made for patients with acute MI or SA or those with atypical chest pain and MPV may be helpful in dividing the patients and making the diagnosis of IHD.

Therefore, the current study was carried out to compare MPV between MI patients and control group (normal coronary angiography results) among patients admitted to Imam Ali Hospital.

\section{Materials and Methods}

This was a descriptive-analytical study. The sample consisted of 200 patients who presented to our university hospital with chest pain. They were divided into five groups considering clinical symptoms, standard diagnostic criteria, electrocardiogram (ECG) changes, cardiac biomarkers, and coronary artery angiography reports. The groups included 40 patients in NSTEMI (typical chest pain, depressed ST segment, positive CK-MB and troponin), 40 patients in UA group (based on criteria of Braunwald and angina during rest in the past 48 hours and progressive angina without accelerating non-cardiac factors), 40 patients with SA (based on history and typical chest pain with history of chronic angina pectoris and some degree of coronary arteries stenosis), 40 patients with STEMI (history of typical chest pain, sudden compression retrosternal pain, ST elevation in two or more leads, hypokinesia on cardiac echocardiography, positive cardiac biomarkers (i.e., CK-MB) and troponin in two assays 6 hours apart), and 40 patients with normal coronary angiography (those with chest pain but without pathologic ECG changes, echocardiography result, or coronary artery angiography). Other variables recorded included FBS (fasting blood sugar), serum cholesterol, MPV, platelet count, WBC count, age, gender, history of cigarette smoking, hypertension, and ejection fraction of the left ventricle (LVEF).

Platelet count and indices were assayed during the first hour after admission to hospital by a sample obtained from venous blood in K-EDTA solution using SYSMEX K-X21.

Patients who had conditions with increased MPV (like high turnover, Bernard-Soulier syndrome, megaloblastic anemia, idiopathic thrombocytopenia, and platelet destruction) and those with decreased MPV (like Big Spleen syndrome, sepsis, and aplastic anemia) were excluded.

By a confidence coefficient of $95 \%$ and using SPSS software (ver. 18.0), the data were analyzed and were expressed by descriptive indices such as mean and standard deviation (SD).

\section{Results}

In 200 studied patients, there were 131 males (65.5\%) and 69 females (34.5\%). There were 55 patients (27.5\%) with diabetes mellitus (DM). Ninety patients (45\%) had hypertension and 56 patients (28\%) had dyslipidemia. Sixty-three patients (31.5\%) were smokers. Of 200 cases, 80 patients (40\%) had STEMI or non-STEMI and coronary angiography was not done. For 120 patients, coronary angiography was done; 40 cases had normal angiography results. Eighty patients had various degrees of coronary stenosis (SA and UA). Seven patients $(8.75 \%)$ had minimal disease, 12 patients $(15 \%)$ had mild disease, 16 cases $(20 \%)$ had single-vessel disease (SVD), 23 cases $(28.75 \%)$ had two-vessel disease (2 VD), 19 cases $(23.75 \%)$ had three-vessel disease (3VD), two patients $(2.5 \%)$ had 3 VD\&LM, and one case (1.2\%) had diffuse CAD. 
MPV was 9.6950 with SD of 0.9857 (Table 1). Other descriptive values are presented in Table 1.

Table 1. Descriptive indices of fasting blood sugar (FBS), mean platelet volume (MPV), platelet count, WBC count, and left ventricular ejection fraction (LVEF)

\begin{tabular}{llllllll}
\hline & Minimum & Maximum & Median & Mean & SD & Upper bound & Lower bound \\
\hline Age & 17 & 88 & 58 & 58.39 & 12.27 & 60.11 & 56.88 \\
FBS & 60 & 294 & 90 & 100.14 & 37.71 & 105.40 & 94.88 \\
MPV & 7.90 & 13.50 & 9.6 & 9.6950 & 0.9857 & 9.83 & 9.55 \\
Platelet count & 22,000 & 430,000 & 222,000 & 251,170 & 295,686 & $2,924,400$ & 209,940 \\
WBC count & 2,100 & 33,000 & 8,100 & 8,883 & 3737.40 & 9404.14 & 8381.86 \\
LVEF & 0.16 & 0.6 & 0.432 & 0.489 & 0.09072 & 0.51 & 0.42 \\
\hline
\end{tabular}

There was no significant difference regarding presence or absence of CAD conventional risk factors (Table 2). Also, no significant difference was seen between gender and MPV $(\mathrm{P}>0.05)$.

Table 2. Comparison of mean platelet volume (MPV) based on major coronary artery disease (CAD) risk factors (hypertension, diabetes mellitus, cholesterol, and cigarette smoking)

\begin{tabular}{|c|c|c|c|c|c|c|c|c|c|c|c|}
\hline & & & No. & Min. & Max. & Mean & SD & $\begin{array}{l}\text { Lower } \\
\text { bound }\end{array}$ & $\begin{array}{l}\text { Upper } \\
\text { bound }\end{array}$ & T-test F & Sig. \\
\hline \multirow{10}{*}{ MPV } & \multirow{2}{*}{ Hypertension } & Yes & 90 & 8.00 & 13.50 & 9.76 & 1.022 & 9.54 & 9.97 & $\mathrm{t} \quad(198$ & \multirow{2}{*}{0.392} \\
\hline & & No & 110 & 7.90 & 12.60 & 9.64 & 0.956 & 9.46 & 9.82 & $0.975)=0.857$ & \\
\hline & \multirow{2}{*}{ Diabetes mellitus } & Yes & 55 & 8.10 & 11.70 & 9.85 & 0.85 & 9.61 & 10.08 & $\mathrm{t} \quad(198$ & \multirow[t]{2}{*}{0.169} \\
\hline & & No & 145 & 7.90 & 13.50 & 9.63 & 1.02 & 9.46 & 9.80 & $0.975)=1.381$ & \\
\hline & \multirow{2}{*}{ Dyslipidemia } & Yes & 56 & 7.90 & 12.50 & 9.74 & 0.971 & 9.48 & 10.003 & $\mathrm{t} \quad(198$ & \multirow{2}{*}{0.670} \\
\hline & & No & 144 & 7.90 & 13.5 & 9.67 & 0.993 & 9.51 & 9.84 & $0.975)=0.427$ & \\
\hline & \multirow{2}{*}{ Smoking } & Yes & 63 & 8.10 & 11.90 & 9.66 & 0.91 & 9.43 & 9.89 & $\mathrm{t} \quad(198$ & \multirow[t]{2}{*}{0.737} \\
\hline & & No & 137 & 7.90 & 13.50 & 9.71 & 1.01 & 9.53 & 9.88 & $0.975)=0.337$ & \\
\hline & \multirow{2}{*}{ Gender } & M & 131 & 7.9 & 13.5 & 9.68 & 1.02 & 9.47 & 9.90 & \multirow{2}{*}{-0.142} & \multirow{2}{*}{0.887} \\
\hline & & $\mathrm{F}$ & 69 & 8 & 12.30 & 9.70 & 0.92 & 9.41 & 9.96 & & \\
\hline
\end{tabular}

Sig.= Significance; $M=$ Male; $F=$ female.

Comparison of mean MPV between males and females in non-STEMI group showed that a significant difference existed between two genders $(\mathrm{P}=0.048)$. In other words, mean value of this index was higher in females than in males.

There was no significant difference regarding MPV between the five studied groups (Table 3). Comparison of mean values of MPV between STEMI, NSTEMI, SA, UA, and control groups (Table 3). Two-by-two comparisons of MPV between minimal, mild, SVD, 2VD, 3 VD. 
Table 3. Comparison of mean platelet values in ST-elevation MI, non-STEMI, stable angina (SA), unstable angina (UA), and control group

\begin{tabular}{lllllllll}
\hline & No. & Mean & SD & Lower bound & Upper bound & ANOVA F & P value \\
\hline \multirow{4}{*}{ MPV } & UA & 40 & 9.68 & 0.98 & 9.36 & 9.99 & & \\
& SA & 40 & 9.45 & 0.75 & 9.21 & 9.69 & & \\
& STEMI & 40 & 9.05 & 1.02 & 9.32 & 9.98 & F $(41.95)=1.046$ & 0.385 \\
& NSTEMI & 40 & 9.85 & 0.86 & 9.57 & 9.43 & & \\
& Control & 40 & 9.82 & 1.23 & 9.43 & 10.22 & & \\
\hline
\end{tabular}

$\mathrm{ANOVA}=$ analysis of variance; $\mathrm{SD}=$ standard deviation .

Diffuse CAD with control group did not show any significant difference (Table 4).

Table 4. Two-by-two comparisons regarding mean platelet volume (MPV) between ST-segment elevation myocardial infarction (MI), NSTEMI, unstable angina (UA), stable angina (SA) with control group

\begin{tabular}{|c|c|c|c|c|c|c|c|c|}
\hline & & No. & $\begin{array}{l}\text { Mean } \\
\text { difference }\end{array}$ & $\begin{array}{l}\text { SD } \\
\text { difference }\end{array}$ & $\begin{array}{l}\text { Lower bound } \\
\text { difference }\end{array}$ & $\begin{array}{l}\text { Upper bound } \\
\text { difference }\end{array}$ & $\mathrm{T}$ test $\mathrm{F}$ & $\begin{array}{l}\mathrm{P} \\
\text { value }\end{array}$ \\
\hline \multirow{4}{*}{ MPV } & UA \& control & 40 & -0.145 & 0.249 & -0.641 & 0.351 & $\begin{array}{l}\mathrm{t}(78,0.975)=- \\
0.582\end{array}$ & 0.563 \\
\hline & SA \& control & 40 & -0.37 & 0.22 & -0.82 & 0.083 & $\begin{array}{l}\mathrm{t}(78,0.975)=- \\
1.63\end{array}$ & 0.107 \\
\hline & $\begin{array}{l}\text { STEMI \& } \\
\text { control }\end{array}$ & 40 & -0.17 & 0.253 & -0.675 & 0.335 & $\begin{array}{l}\mathrm{t}(78,0.975)=- \\
0.67\end{array}$ & 0.505 \\
\hline & $\begin{array}{l}\text { NSTEMI \& } \\
\text { control }\end{array}$ & 40 & 0.025 & 0.237 & -0.449 & 0.499 & $\begin{array}{l}\mathrm{t} \quad(78,0.975)= \\
0.105\end{array}$ & 0.917 \\
\hline
\end{tabular}

$\mathrm{SD}=$ standard deviation.

Also, there was no significant difference regarding MPV between patients with MI who were diabetic and those who were not diabetic $(\mathrm{P}=0.281)$.

\section{Discussion}

This study was done with the objective of comparing MPV between MI patients with those who had normal coronary angiography results.

Regarding the important role that platelets have in atherosclerosis and instability of atherosclerotic plaques and platelet plug formation and subsequent coronary artery stenosis, several studies have been done in this field and some of them have shown more activity in larger platelets (high MPV) compared to smaller platelets.

In a study in 2005 on 200 patients, MPV was compared between STEMI, NSTEMI, UA, SA, and control groups. The results showed that MPV was higher in MI group compared to SA and control group. The authors suggested MPV as an independent risk factor for MI (Kiliçli-Camur Netal, 2005). These results are in contrast to our results.

In another study in 2008, MPV was compared between a group of 216 patients with ACS with a group of 120 patients with SA. MPV in ACS group was $10.4 \mathrm{fL}$ and this was $8.90 \mathrm{fL}$ in SA group which the difference was statistically significant (Yilmaze MBetal, 2008). This study also contradicts our findings.

In our study, MPV was compared between four 40-patient groups, after controlling for confounding variables, revealed values of $9.85 \mathrm{fL}$ in NSTEMI, $9.68 \mathrm{fL}$ in UA, $9.45 \mathrm{fL}$ in SA, $9.65 \mathrm{fL}$ in STEMI, and $9.82 \mathrm{fL}$ in control group. There was no significant difference at different age groups and genders which contradict Yilmaze study (Yilmaze et al, 2008).

MPV was only compared between males and females with NSTEMI. In males, this was $9.62 \mathrm{fL}$ and in females this was $10.16 \mathrm{fL}$, a significant difference $(\mathrm{P}=0.048)$. 
In a study in 2009 (Ranjh et al., 2009), platelet count in ACS group $\left(210 \times 10^{3}\right)$ was lower compared to SA group $\left(276 \times 10^{3}\right)$ and control group $\left(256 \times 10^{3}\right)$. However, MPV in ACS group $(10.93 \mathrm{fL})$ was higher than in control group (10.3 fL). In our study, platelet count was 256,500 in NSTEMI group, 323,000 in UA group, 323,000 in SA, 228,380 in STEMI group, and 234,420 in control group. No difference was seen between ACS, SA, and control groups. This result is not in agreement with Ranjh study (Ranjh et al., 2009).

In another study in 2010 (Tavil, Sen, \& Yazici, 2010), MPV in diabetics who had MI was $10.93 \mathrm{fL}$, but MPV was 9.12 in normal non-diabetic patients, a significant difference. In our study, of 200 patients included, 80 patients had MI in which 28 cases were diabetics. These were compared to 31 non-diabetic patients with normal coronary angiography results. In diabetics, MPV was $9.87 \mathrm{fL}$ and in diabetics this was $10.09 \mathrm{fL}$, with no significant difference.

\section{Conclusion}

Considering the results obtained here and other former results, it is understood that platelet count increase or decrease is not seen with IHD and cannot have clear association with atherosclerosis, ischemia, and coronary artery thrombosis. Also, increase in MPV during ischemia may not have a clear role in ischemia and thrombus formation and this is not proven. Even increased MPV is seen, it is not clear whether this increased MPV has a significant role in thrombus formation or initiation. This has not been established in former studies, or in our study.

Although in some conditions such as ITP or platelet destruction, platelet count decreases and in a compensatory action, new immature platelets which are larger in size are released to the circulation. However, this process has not been shown to play role in thrombus formation or plaque rupture and increased MPV does not have any role in thrombus formation.

Also, high MPV has no relationship with mortality in IHD patients. Also there is still a question about the role of high MPV in predicting the outcome of patients. High MPV cannot be used to diagnose IHD patients. It seems that this field needs more research.

\section{Acknowledgments}

This article is based on a thesis submitted to the graduate studies office in partial fulfillment of requirements for the degree of cardiology physician by MasoudJalilian in Kermanshah University of Medical Sciences, Faculty of Medicine.

\section{Conflict of Interest}

The authors declare that there is no conflict of interests regarding the publication of this paper.

\section{References}

Harrison's internal Medicine. (2012). 18th ed. New York: McGaw Hill, p457

Kiliçli-Camur, N., Demirtunç, R., Konuralp, C., Eskiser, A., \& Başaran, Y. (2005).Could Mean Platelet volume be a predictive marker for acute myocardial infraction?. Med SciMonit, 11(8), CR387-92.

Ranjh, M. P., Divya, R., Mehta, V. K., \& Krishnan, M. G. (2009). Significance of platelet volume indices and platelet count in ischaemic heart disease. $J$ Clin Pathol, 62(9), 830-3. http://dx.doi.org/10.1136/Jep.2006.066787

Tavil, Y., Sen, N., \& Yazici, H. (2010). Coronary heart disease is associated with mean platelet volume in type 2 diabetic patients. Platelets, 21(5), 368-72. http://dx.doi.org/10.3109/09537101003628421

Viziolil, L., Muscari, A., \& Muscari, A. (2009). The relationship of mean platelet volume with the risk and

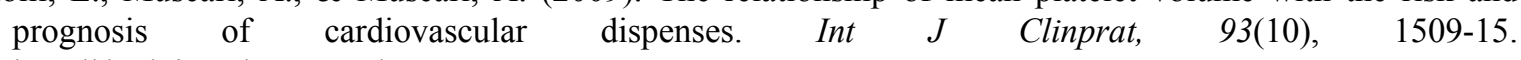
http://dx.doi.org/10.11111/J.1742-1241. 2009. 02070.x

Yilmaze, M. B., Cihan, G., Guray, Y., Gurary, U., Kisacik, H. L., Sasmaz, H., \& Korkmaz, S. ( 2008). Role of mean platelet Volume in triaging acute coronary syndromes. J thrombthrombolysis. 26(1), 4954.

Wintrobe's DICI elinicalHematolog. (2014). Philadelphia: Lippincott\& Willkins, p389, 1047

\section{Copyrights}

Copyright for this article is retained by the author(s), with first publication rights granted to the journal.

This is an open-access article distributed under the terms and conditions of the Creative Commons Attribution license (http://creativecommons.org/licenses/by/3.0/). 